\title{
Surveillance imaging for metastasis in high-risk melanoma: importance in individualized patient care and survivorship
}

\author{
Morganna Freeman ${ }^{1} \&$ Shachar Laks*,2 \\ ${ }^{1}$ Department of Medical Oncology and Therapeutics Research, City of Hope, Duarte, CA 91010, USA \\ ${ }^{2}$ Department of Surgery, East Carolina University, Greenville, NC 27834, USA \\ *Author for correspondence: Tel.: +1 252744 4110; lakss17@ecu.edu
}

\begin{abstract}
Most patients newly diagnosed with melanoma have early-stage disease considered of good prognosis. However, with a risk of recurrence, appropriate follow-up may include surveillance imaging for early relapse detection. Previously, surveillance imaging to detect recurrences was considered unjustified, given the lack of effective treatments. Now, systemic therapies have improved, and patients with low tumor burden may derive benefit from surveillance imaging. Despite this, controversy exists regarding the role of surveillance imaging in early-stage melanoma survivorship, in part reflected by the lack of consensus on specific imaging protocols and broad guidelines. This review discusses published evidence on surveillance imaging to detect metastasis in high-risk melanoma, the need for early recurrence detection and implications for value-based clinical decision-making, survivorship care and multidisciplinary patient management.
\end{abstract}

First draft submitted: 10 February 2019; Accepted for publication: 1 April 2019; Published online: 18 April 2019

Keywords: early detection • imaging • melanoma • surveillance • survivorship

Historically, surveillance imaging to detect metastasis after definitive treatment of early-stage melanoma was considered by many to be futile due to the lack of effective therapeutic interventions for recurrent or metastatic disease $[1,2]$. Until recently, this negatively impacted improvements in survival for melanoma patients whose routine surveillance identified recurrences; thus the inclusion of routine imaging as a part of surveillance plans has been met with controversy $[2,3]$. Likewise, there is a lack of expert consensus and evidence-based guidelines regarding the optimal imaging modalities and schedules to best identify asymptomatic melanoma recurrences, and existing recommendations are not defined for individual melanoma stages despite the clear evidence that relapse risk escalates with higher stage [4-8] (Table 1). Using stage as the sole driver of follow-up intensity is problematic as well, since increasing stage does not necessarily correlate to inherent risk of recurrence of metastasis. As such, existing guidelines are relatively broad, with a wide range of follow-up frequencies and imaging modalities with varying sensitivities and specificities for detection [4-8]. A recent meta-analysis showed that ultrasonography (US) had the highest sensitivity and specificity for lymph node metastasis ( 96 and 99\%, respectively) compared with computed tomography (CT), positron emission tomography (PET) and PET-CT [9]. However for distant metastasis, PET-CT had the highest sensitivity (86\%), which is tempered by its lower specificity, as well as its $33 \%$ positive predictive value in low-risk patients (estimated 5\% recurrence risk). These results may suggest the imaging modality should be selected based on stage and risk of distant metastasis.

Surveillance guidelines are a critical component of survivorship care plans, further highlighting the need for a standardized, risk-based approach. On average, $20-30 \%$ of early stage melanoma patients will develop a recurrence within 5 years [10,11], yet imaging recommendations are generally reserved for patients with Stage IIB melanoma or higher, thus omitting distant metastatic surveillance from a subset of earlier stage patients with a biological propensity for relapse.

Systematic monitoring for metastatic disease is important and relevant given numerous effective, contemporary targeted and immunotherapies for melanoma. These therapies are extending disease-free intervals and overall 


\begin{tabular}{|c|c|c|c|c|}
\hline & AAD (2019) & NCCN (2019) & ESMO (2015) & NICE (2015) \\
\hline Baseline imaging & $\begin{array}{l}\text { - Stage I-II: not recommended } \\
\text { in asymptomatic patients } \\
\text { - Patients with equivocal LN by } \\
\text { exam should have nodal US }\end{array}$ & $\begin{array}{l}\text { - Stage I-II: not recommended } \\
\text { in asymptomatic patients } \\
\text { - Stage IIIA (positive SLNB): } \\
\text { consider baseline imaging } \\
\text { - Stage IIIB-C (positive SLNB): } \\
\text { perform baseline imaging } \\
\text { - Stage III (in-transit, } \\
\text { microsatellites, clinical node): } \\
\text { perform baseline imaging; } \\
\text { consider brain MRI in Stage } \\
\text { IIIC-D } \\
\text { - Patients with equivocal LN by } \\
\text { exam should have nodal US }\end{array}$ & $\begin{array}{l}\text { - pT1a: not recommended } \\
\text { - pT1b-pT3a: nodal US } \\
\text { - pT3b-pT4b: CT or PET scans }\end{array}$ & $\begin{array}{l}\text { - Stage IIC without SLNB, Stage } \\
\text { III, suspected Stage IV: perform } \\
\text { CT imaging } \\
\text { - Suspected Stage IV: brain MRI } \\
\text { - 0-24 years of age with Stage III, } \\
\text { suspected Stage IV: whole body } \\
\text { MRI }\end{array}$ \\
\hline Surveillance imaging & $\begin{array}{l}\text { - Stage I-IIA: not recommended } \\
\text { - Stage IIB-IV: surveillance } \\
\text { imaging for up to } 3-5 \text { years, } \\
\text { based on risk of recurrence and } \\
\text { new primary melanomas } \\
\text { - Forgoing SLNB when eligible, } \\
\text { failed SLNB procedures, } \\
\text { positive SLNB without CLND, } \\
\text { expert nodal US is available: } \\
\text { surveillance US of nodal basin }\end{array}$ & $\begin{array}{l}\text { - Stage I-IIA: not recommended } \\
\text { without symptoms } \\
\text { - Stage IB-IV: consider } \\
\text { surveillance imaging every 3-12 } \\
\text { months for up to 3-5 years } \\
\text { - Forgoing SLNB when eligible, } \\
\text { failed SLNB procedures, } \\
\text { positive SLNB without CLND, } \\
\text { expert nodal US is available: } \\
\text { surveillance US of nodal basin } \\
\text { every 3-12 months for 2-3 years }\end{array}$ & $\begin{array}{l}\text { - Thin melanoma: not } \\
\text { recommended } \\
\text { - High-risk melanoma (thick or } \\
\text { previous metastases): nodal US, } \\
\text { CT, whole body PET or PET/CT } \\
\text { - Consult national guidelines }\end{array}$ & $\begin{array}{l}\text { - Stage I-IIB, IIC with negative } \\
\text { SLNB: not recommended } \\
\text { - Stage IIC without SLNB, Stage } \\
\text { III: consider imaging in a clinical } \\
\text { trial or every } 6 \text { months for } 3 \text { years } \\
\text { (with policy and funding) } \\
\text { - Stage IV: offer personalized } \\
\text { schedule }\end{array}$ \\
\hline
\end{tabular}

AAD: American Academy of Dermatology; CLND: Completion lymph node dissection; CT: Computed tomography; ESMO: European Society for Melanoma Oncology; LN: Lymph node; MRI: Magnetic resonance imaging; NCCN: National Comprehensive Cancer Network; NICE: National Institute for Health and Care Excellence; PET: Positron emission tomography; US: Ultrasonography; SLNB: Sentinel lymph node biopsy.

survival, and nearly all modern melanoma therapies elicit improved responses and outcomes with lower metastatic tumor burden [12-17]. It remains to be shown whether the combination of surveillance imaging and subsequent early therapeutic intervention directly results in improved clinical outcomes, in part due to effective therapies being relatively new and the complexities and potential costs associated with a randomized, prospective trial that would, conceptually, exclude some high-risk patients from surveillance imaging. However, there are data to support the utility of surveillance imaging to identify clinically occult, asymptomatic melanoma recurrences, though a direct survival benefit of early detection has not been demonstrated [18]. Together with the prognostic value of lower metastatic tumor burden upon recurrence, these findings are germane to routine use of surveillance imaging in melanoma patients at a high risk for recurrence. To foster that consideration, we discuss the published evidence reporting melanoma recurrence detection rates with routine imaging (Table 2) and the implications for early detection in the context of contemporary therapies, as well as the current controversies surrounding routine imaging for early stage disease.

\section{Benefits of early detection on curative-intent resections \& survival}

A large prospective study in Germany was one of the first to suggest that earlier detection of melanoma recurrences conferred a survival benefit, even when accounting for lead time bias. Enrolled patients were followed regularly with quarterly history and physical exam, as well as stage-specific blood work and imaging (ultrasound and chest x-ray) [19]. Patients with melanoma $>1 \mathrm{~mm}$ thick routinely underwent sentinel lymph node biopsy [20]. Within 2 years, 112/1969 patients with Stage I-III melanoma recurred (3.6\% were Stage IA, 10.7\% were Stage IB, 19.6\% were Stage IIA, $8.9 \%$ were Stage IIB, 16.1\% were Stage IIIA and $41.1 \%$ were Stage IIIB by 2002 AJCC staging at the time of study enrollment) [20]. 46\% (93/204) of metastases were detected by imaging and 50\% (101/204) were detected by physicians during follow-up [19]. Metastases were classified as being discovered 'early' (organ/lymph node tumor size $\leq 2 \mathrm{~cm}$, more than five involved nodes [more than ten for in-transit melanoma], and/or eligible for curative-intent surgery) or 'late' (metastases not meeting those criteria). In a follow-up report, lead-time bias was accounted for using sojourn time, the estimated amount of time for a clinically occult recurrence to manifest as clinically detectable [20]. The ten-year overall survival rates after adjusting for lead-time bias were $40.5 \%$ for early-detection and $25.6 \%$ for late-detection $(\mathrm{p}=0.02)$. These reported survival rates are higher than would be expected without effective systemic treatments, which may reflect more locoregional recurrences being earlydetected. However, there was also a significant survival benefit with early detection when locoregional recurrences 


\begin{tabular}{|c|c|c|c|c|c|}
\hline Study and design & Stage & $\begin{array}{l}\text { Surveillance imaging } \\
\text { regimen }\end{array}$ & $\begin{array}{l}\% \text { of total recurrences } \\
\text { identified by imaging }\end{array}$ & $\begin{array}{l}\% \text { of distant recurrences } \\
\text { identified by imaging }\end{array}$ & Ref. \\
\hline $\begin{array}{l}\text { Garbe et al. (2003) } \\
\text { prospective, } \\
\text { multi-center } \\
n=2008\end{array}$ & I-III & $\begin{array}{l}\text { Abdominal U/S, chest } \\
\text { x-ray and nodal basin U/S } \\
\text { annually for Stage I-II or } \\
\text { bi-annually for Stage III }\end{array}$ & $47 \%$ & $\mathrm{~N} / \mathrm{R}$ & [19] \\
\hline $\begin{array}{l}\text { Podlipnik et al. (2016) } \\
\text { prospective, single center } \\
n=290\end{array}$ & IIB-III & $\begin{array}{l}\text { Whole body CT and brain } \\
\text { MRI bi-annually for } 5 \\
\text { years, then chest } x \text {-ray for } \\
\text { years } 5-10\end{array}$ & $56.7 \%$ & $83.6 \%$ & [23] \\
\hline $\begin{array}{l}\text { Park et al. (2017) } \\
\text { retrospective, single } \\
\text { center } \\
n=466\end{array}$ & II-IV (resected) & $\begin{array}{l}\text { CT scan at least } \\
\text { biannually for } 2 \text { years and } \\
\text { then annual for } 3 \text { years }\end{array}$ & $59 \%$ & $75 \%$ & [24] \\
\hline $\begin{array}{l}\text { Lim et al. (2018) } \\
\text { retrospective, single } \\
\text { center } \\
n=173\end{array}$ & IIB-III & $\begin{array}{l}\mathrm{CT} \mathrm{C} / \mathrm{A} / \mathrm{P} \text { or } \mathrm{PET} / \mathrm{CT} \text {, brain } \\
\mathrm{MRI} \text { biannually for } 3 \text { years } \\
\text { and then annual up to } 5 \\
\text { years }\end{array}$ & $66 \%$ & $\mathrm{~N} / \mathrm{R}$ & [25] \\
\hline $\begin{array}{l}\text { Lewin et al. (2018) } \\
\text { retrospective, single } \\
\text { center } \\
n=170\end{array}$ & III & $\begin{array}{l}\text { Biannual PET/CT at least } \\
\text { twice and up to } 2 \text { years; } \\
\text { brain MRI at } 6 \text { and } 12 \\
\text { months for Stage IIIC }\end{array}$ & $70 \%$ & $\mathrm{~N} / \mathrm{R}$ & [22] \\
\hline $\begin{array}{l}\text { Livingstone et al. (2015) } \\
\text { prospective, multi-center } \\
\mathrm{n}=668\end{array}$ & $0-I V$ & $\begin{array}{l}\text { Quarterly (35\%) } \\
\text { Bi-annual }(40 \%) \\
\text { Annual }(16 \%)\end{array}$ & $77 \%$ & $96.5 \%$ & [26] \\
\hline $\begin{array}{l}\text { Leon-Ferre et al. (2017) } \\
\text { retrospective, } \\
\text { single-center } \\
n=299\end{array}$ & III-IV (resected) & $\begin{array}{l}\text { At least one PET/CT as } \\
\text { part of surveillance } \\
\text { within } 1 \text { year of definitive } \\
\text { surgery }\end{array}$ & $60 \%$ & $\mathrm{~N} / \mathrm{R}$ & [21] \\
\hline $\begin{array}{l}\text { Madu et al. (2017) } \\
\text { prospective, single-center } \\
n=18\end{array}$ & IIIB-IIIC & $\begin{array}{l}\mathrm{PET} / \mathrm{CT} \text { biannually for } 2 \\
\text { years }\end{array}$ & $89 \%$ & $\mathrm{~N} / \mathrm{R}$ & [27] \\
\hline $\begin{array}{l}\text { Romano et al. (2010) } \\
\text { retrospective, } \\
\text { single-center } \\
\mathrm{n}=340\end{array}$ & III & $\begin{array}{l}\text { Typically CT scans at } \\
\text { quarterly to biannual } \\
\text { clinical visits but no } \\
\text { standard regimen }\end{array}$ & $\mathrm{N} / \mathrm{R}$ & $53 \%$ & [30] \\
\hline $\begin{array}{l}\text { Lee et al. (2016) } \\
\text { retrospective, } \\
\text { single-center } \\
n=738\end{array}$ & II & $\begin{array}{l}\text { Imaging performed at } \\
\text { discretion of physician }\end{array}$ & $\mathrm{N} / \mathrm{R}$ & $47 \%$ & [29] \\
\hline $\begin{array}{l}\text { Berger et al. (2017) } \\
\text { retrospective, two centers } \\
n=581\end{array}$ & II & $\begin{array}{l}\text { Imaging performed at } \\
\text { discretion of physician }\end{array}$ & $\begin{array}{l}21 \% \\
50 \%(\text { Stage IIA) }\end{array}$ & $\mathrm{N} / \mathrm{R}$ & [28] \\
\hline
\end{tabular}

were evaluated separately. While this study did not show that surveillance confers a survival benefit, the results support the importance of early detection.

Depending on degree of tumor burden and metastatic site, some patients with nodal and oligometastatic disease can be treated with curative-intent surgery. Curative-intent salvage therapy (primary resection) was shown to have a survival benefit compared with salvage therapy without curative intent in a retrospective study at the Mayo Clinic. The study evaluated 299 patients with Stage III-IV melanoma who had at least one PET/CT for surveillance within a year of definitive surgery. Recurrences were classified as clinically occult if detected by imaging and clinically evident if symptomatic. Survival outcomes were compared for patients with recurrences treated by curative intent versus non-curative-intent salvage therapy [21]. Imaging identified 60\% (98/162) of recurrences, and 66\% (65/98) of those clinically occult recurrences were treated with curative-intent salvage therapy. While not significant $(\mathrm{p}=0.051), 48 \%$ of clinically occult visceral metastases were treated with curative-intent salvage therapy compared with $20 \%$ of clinically evident visceral metastases. Curative-intent surgery was associated with statistically improved survival compared with salvage therapy without curative intent for both clinically occult and clinically evident recurrences. Taking together the benefit of curative-intent surgery and the asymptomatic detection of $60 \%$ 
of the first recurrences by imaging, the authors suggest that history, physical exams and surveillance imaging may be useful in combination to identify recurrences that can be treated with curative intent salvage therapy.

Another study in which 170 Stage III patients had substage-specific routine imaging that included PET/CT at least twice at six-month intervals found that PET/CT identified asymptomatic recurrences in $69 \%(45 / 65)$ of those who relapsed [22]. Of the 65 patients who recurred, 51\% (33/65) underwent curative-intent resection, including approximately half of the patients with PET-detected recurrences. Ten of the patients who had curativeintent resection were disease-free at a median of 24 months of follow-up and 70\% (7/10) of these patients had PET-detected recurrences.

\section{Efficacy of routine cross-sectional imaging in detecting asymptomatic distant recurrences}

Other recent studies employing CT as the primary imaging method have shown higher rates of recurrence detection, particularly those that are distant. A 10-year prospective study prescribed bi-annual total body CT and brain MRI surveillance for 290 Stage IIB-IIIC melanoma patients during years one through five, followed by annual chest x-ray during years 5 through 10 [23]. With a median follow-up of 2.5 years, 39\% (115/290) of patients developed recurrences and $56.7 \%$ of these were detected by imaging, which proved to be the most effective method of detection irrespective of AJCC stage. Imaging identified $84 \%(51 / 61)$ of distant metastases, $92 \%$ of which were asymptomatic. Given that $50 \%$ of the recurrences were detected by CT alone and CT detected metastases consistently throughout the first five years of the study, the authors recommended the use of CT in the first five years of follow-up for high-risk melanoma patients.

Similarly, investigators from the National Cancer Institute recommended routine cross-sectional imaging for the surveillance of high-risk, asymptomatic melanoma patients based on their analysis of CT screening in four adjuvant vaccine trials [24]. The 466 patients evaluated were primarily Stage II (25\%) and Stage III (70\%) and received CT scans at least every 6 months for the first two years and then annually up to year 5 . In the 5-year study period, $48 \%(225 / 466)$ of patients recurred and $59 \%(131 / 225)$ of these recurrences were detected by CT. $48 \%(26 / 54)$ of Stage II recurrences and 68\% (108/158) of Stage III patients recurrences were systemic, and 75\% (107/144) of all systemic recurrences were identified by imaging while the patient was asymptomatic [24].

A third group of investigators corroborated the important role of imaging in detecting subclinical melanoma recurrences, particularly those amenable to surgery [25]. In a retrospective study between 2013 and 2015 in the United Kingdom, 173 Stage IIIB-IIIC patients were recommended to have a CT of the chest/abdomen/pelvis or PET/CT, plus brain MRI at baseline and then every 6 months for 3 years and annually up to 5 years. After a median follow-up of almost 2 years, $47.4 \%$ (82/173) of patients recurred, with a median time to recurrence of 10.1 months, and $68.2 \%(56 / 82)$ of these recurrences were distant. $66 \%$ of patients had asymptomatic recurrences detected upon imaging $(54 / 82)$. The majority $(72 / 82 ; 88 \%)$ of patients went to either surgery $(37 / 72 ; 51 \%)$ or systemic treatment $(33 / 82 ; 46 \%)$. Median overall survival with systemic therapy was 12.9 months, but most patients had BRAF inhibitor monotherapy or ipiliimumab, which were later found to be inferior to BRAF combination therapy and anti-PD-1 therapy, respectively. Median overall survival rate was not reached for patients who underwent surgery, and $80 \%$ of those who underwent resection were alive after 2 years. Survival outcomes with surgery for Stage III versus Stage IV relapses were not significantly different. These findings underscore the utility of imaging to detect asymptomatic recurrences and the continued importance of surgery for appropriately selected patients who have low volume recurrent disease detected by imaging.

The imaging detection rate of distant recurrences has been reported to be as high as $96.5 \%$ in a large, prospective German registry study of 668 patients [26] and a recent small pilot study of bi-annual PET/CT in 18 Stage IIIB-C melanoma patients also found that the sensitivity of PET-CT for asymptomatic recurrence detection was $91 \%$ [27]. There are limitations to some of the studies described above, including short follow-up and small cohorts, as well a lack of demonstrated survival benefit with surveillance and early detection. However, two important points regarding routine surveillance imaging can be drawn from the aforementioned studies. First, imaging alone is effective at detecting the majority of distant recurrences. Second, the majority of recurrences (92-100\%) identified by imaging are detected while patients are asymptomatic, which generally portends a lower disease burden and eligibility for curative surgery, which could be beneficial for patient outcomes.

\section{Patient detection versus imaging}

Some have questioned the role of routine imaging in early stage melanoma surveillance, citing evidence that recurrences are more frequently patient-detected (40-59\%) compared with detection by imaging (20-32\%) [28-30]. 
However, these studies must be interpreted with caution regarding imaging. Imaging can only be truly effective if it is utilized routinely in the surveillance program of the study, and sensitivity for melanoma recurrence varies between nodal basin US, chest x-ray, CT scan and PET [2]. Notably, in two of the aforementioned studies in which patients received follow-up imaging at regular intervals, $17-27 \%$ of recurrences were patient-detected, while $57-59 \%$ of recurrences were imaging-detected [23,24].

If an imaging schedule is not routine, is unknown, or not employed, then the primary means of recurrence detection will have to be by physicians or patients. As an example, in a joint study of 171 Stage II patients who experienced recurrence, imaging was largely left to the discretion of the treating physicians [28]. The authors point out that there was no consistent follow-up or imaging schedule with numerous physicians involved across two institutions and some patients were referred from outside practices. In this study, $40 \%$ of recurrences were detected by patients and $30 \%$ were detected by physicians. However, imaging was comparatively the most successful method to detect recurrences in Stage IIA patients [28]. Current guidelines do not recommend surveillance imaging for Stage IIA patients in the absence of signs or symptoms [4-8].

Skin and subcutaneous metastases are logically more likely to be detected by patients, while imaging is more useful to detect clinically occult distant and visceral metastases, which would otherwise go unnoticed until symptomatic. A retrospective study at Memorial Sloan Kettering of 219 Stage II patients who experienced relapse found that imaging detected only $2 \%$ of local and in-transit recurrences, while the majority were detected by the patient (62\%) or physician (36\%) [29]. However, imaging was much more effective at identifying visceral recurrences. Even though the use of surveillance imaging was left to the discretion of the treating physician, it still detected $47 \%$ of systemic recurrences, all asymptomatic [29]. Similar findings were reported for 340 Stage III patients who experienced relapse at the same center, in which $62.5 \%$ of local/in-transit recurrences were identified by the patient or family compared with $1 \%$ by imaging, while $53 \%$ of visceral recurrences were found by imaging, which was 'typically' done prior to regular follow-up visits [30]. Taken together, optimal surveillance of early stage melanoma patients should include patient education on regular skin and nodal self-examinations, clinical examinations with physicians and routine imaging.

The role for imaging in early identification of metastatic disease to improve patient outcomes As summarized in Table 2 and Figure 1, the studies discussed herein have shown that imaging is an important tool to identify melanoma recurrences, particularly those that are distant and asymptomatic, even when the specific modality and frequency of imaging were not uniform. Ultimately, the purpose of surveillance imaging is to aid in early detection and identify asymptomatic recurrences amenable to immediate treatment while tumor burden is limited, which may positively impact the survival of melanoma patients. While there are distinct differences in treatment options if a patient presents with locally advanced disease versus bulky or disseminated disease, contemporary therapies, including oncolytic talimogene laherparepvec (T-VEC) [13]; checkpoint inhibition with ipilimumab [16], pembrolizumab [12,17,31] and nivolumab [32]; and targeted therapy with dabrafenib and trametinib [14,33,34] and vemurafenib and cobimetinib [35] have all shown improved responses and/or survival outcomes in subclinical disease and/or when the melanoma tumor burden is low. As discussed above, the potential for curative-intent resection, which is also associated with improved survival, is aided by using imaging to detect asymptomatic recurrences $[21,36]$.

\section{Targeting surveillance imaging toward high-risk melanoma patients}

Of course, routine imaging is not appropriate for all melanoma patients. The overuse of intensive imaging in low-risk patients represents an inefficient use of healthcare resources [26] and may result in unnecessary radiation exposure for the patient. A melanoma diagnosis has profound emotional and psychological effects on patients, including general anxiety at diagnosis, fear of recurrence or of a new cancer, concerns about surgical scars and social isolation from avoiding outdoor activities involving sun exposure [37]. Few studies have evaluated the psychological effects of follow-up care, however one noted that regular follow-up may help patients cope with the idea of recurrence and offer opportunities for patient education [38]. Some patients may demand certain tests or studies in hopes of earlier detection and better outcome or to ease anxiety [39]. This may go against the clinician's better judgment, therefore it is critical to tailor surveillance according to individual risk, keeping in mind that there is evidence to support radiographic surveillance in early stage patients as noted above. For patients whose high-risk melanoma necessitates surveillance imaging, scan-associated anxiety is a concern and has not been well-studied in melanoma. One study evaluated quality of life in patients with clinical Stage III melanoma who underwent cross-sectional 


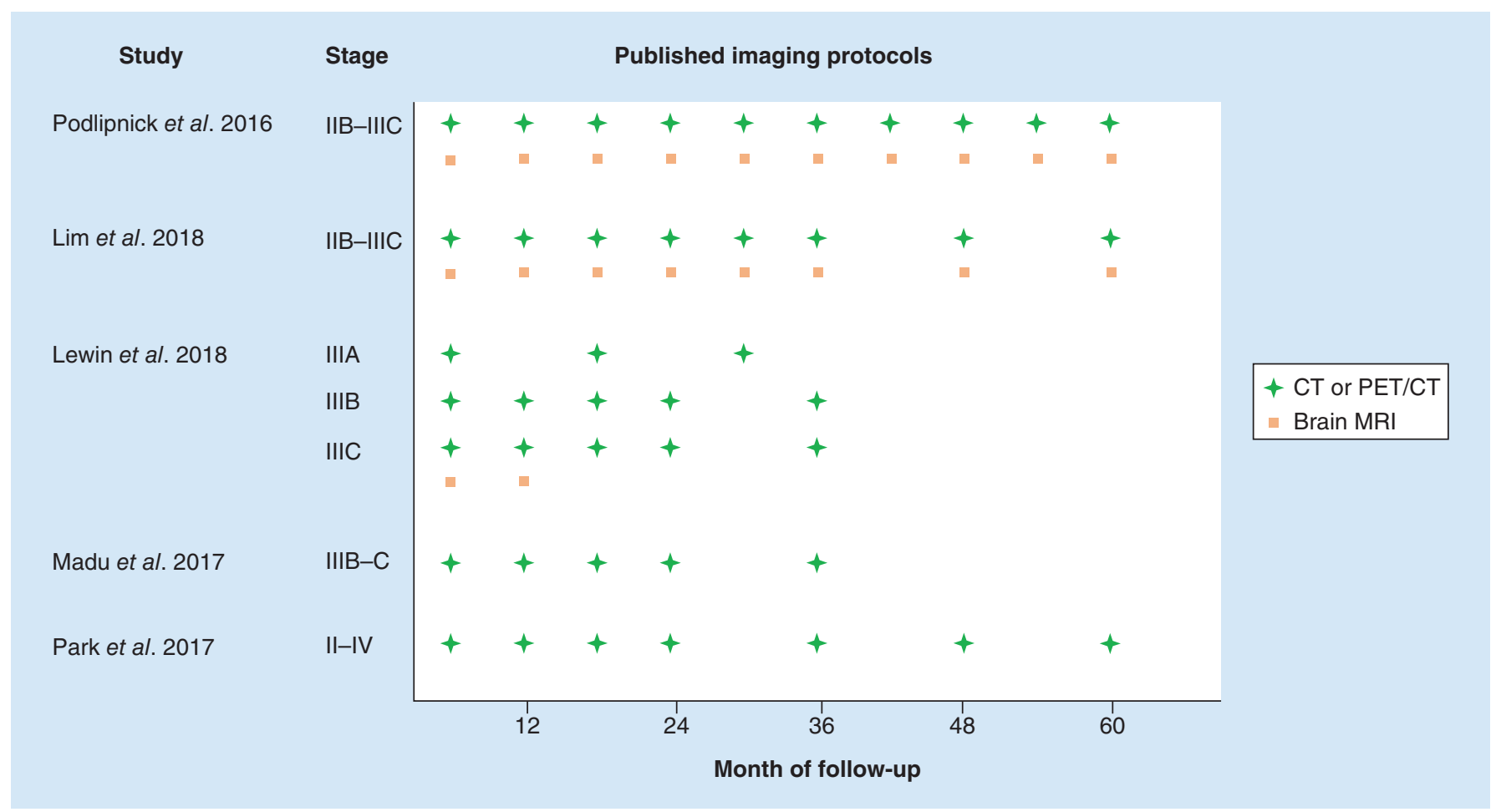

Figure 1. Published imaging protocols to detect recurrences in high-risk patients.

The schematic summarizes published imaging protocols used for metastatic screening after definitive surgery in melanoma patients at a high risk of recurrence according to clinicopathologic staging. The sensitivities of these protocols for recurrence detection are listed in Table 2. CT or PET/CT is indicated by a green cross and brain MRI by an orange square.

CT: Computed tomography; PT: Positron emission tomography; MRI: Magnetic resonance imaging.

surveillance imaging and found that the overall patient-reported burden of imaging was low despite some feelings of embarrassment, anxiety and discomfort [40].

The imaging schedules used by the reviewed studies are depicted in Figure 1. Each study had a different design, patient cohort and follow-up time, making an overarching recommendation on surveillance imaging from these studies alone difficult. Furthermore, earlier stage patients with adverse tumor biology and/or clinical histories were not included and will be important for future studies. However, across the studies, patients had at least three CT or PET/CT scans during the first 3 years of follow-up, with most studies employing biannual imaging during this time (thus six scans, excluding brain imaging). A systematic review and imaging studies that include high-risk earlier stage patients may be helpful to better define imaging recommendations in the future. Additionally, while focus of our literature review is on melanoma progression after definitive resection of the primary tumor, it should also be noted that whole-body imaging to monitor for new primary melanomas can be useful for patients with a personal or family history of melanoma or at high risk for melanoma due to nevus count and/or dysplastic nevi [41].

\section{Future directions for surveillance \& survivorship}

Molecular and genetic profiling continues to play a key role in individual patient risk assessment for many malignancies, with emerging use in melanoma. Genetic profiling in uveal melanoma has led to risk-adjusted imaging surveillance plans that have been readily adopted and are reflected in recent national guidelines [42]. Intensive surveillance is focused on patients with biologically aggressive tumors as ascertained by molecular prognostication, while low-risk patients have less frequent and intense follow-up for metastatic screening [43,44]. Genetic risk profiling is also used for risk stratification in cutaneous melanoma, with recent evidence that it is starting to be incorporated into clinical decision-making including intensity of follow-up and referral patterns [45-49].

As the science and understanding of melanoma continue to evolve, so should the best avenues for risk assessment. The current state of science in cutaneous melanoma relies on both traditional tools (pathologic staging characteristics), as well as genetic and molecular profiling [50-54], wherein $B R A F$ mutation status now has predictive 
and prognostic implications for patients with resected early stage disease [55,56]. There may be a role for both in cutaneous melanoma to provide comprehensive risk assessment in order to accurately identify patients who are unlikely to recur, thus best managed primarily with regular skin and nodal examinations and likely to recur, thus best managed with the addition of surveillance imaging into their follow up care and survivorship plan. Recent studies have shown the utility [50] and early adoption of this combinatorial, personalized approach to impact melanoma patient management $[46,47]$. However given the rising incidence of melanoma and the changing treatment landscape, which includes targeted and immunotherapies for resected and unresectable Stage III-IV disease, restaging with AJCC v8 and paradigm shifts in completion lymphadenectomy, our surveillance recommendations must similarly evolve to account for individual risk and therapeutic benefit of low metastatic tumor burden. To clearly understand the impact of risk-tailored surveillance protocols on survival outcomes there is an as-yet unmet need for prospective, randomized clinical trials to evaluate different surveillance regimens according to clinicopathologic and genetic/molecular prognostic factors. As mentioned before, a randomized trial of surveillance imaging versus no imaging could withhold imaging from patients at a high risk of recurrence. Thus, comparisons of frequency and intensity of imaging regimens may be more appropriate and add precision to the broad recommendations currently in place.

Keeping in mind the costs associated with diagnostic studies, value-based clinical decision making is also an essential part of risk-based surveillance. Two decades ago it was reported that recurrence screening in melanoma survivors accounted for an estimated $80 \%$ of care costs [57], accounting for millions invested to provide effective 10-year surveillance. Compared with routine skin exams, CT and PET-based imaging is costly and should be used judiciously based on a patient's estimated risk of relapse [58]. Comprehensive risk assessment tools that combine staging, clinical factors and genomic prognostication may represent a cost-effective clinical practice guideline for post-treatment melanoma surveillance, arguing further for the need to strengthen current clinical evidence regarding appropriate intervals for follow-up based on risk of recurrence.

\section{Conclusion}

As noted above, routine surveillance imaging for asymptomatic melanoma metastases has a clear role from interventional and overall survival perspectives in select patients, and traditionally that selection was based upon AJCC stage. With changes in AJCC staging, management away from completion lymphadenectomy and adjuvant treatment, a new need is emerging for comprehensive risk stratification and risk-based surveillance planning. As the incidence of melanoma continues to rise while mortality rates remain stable, there is a growing population of melanoma survivors, many of whom receive follow-up care in both primary and secondary care settings. Considering the psychosocial, financial and disease-free survival impacts such a surveillance plan would have, clear, consistent guidelines should be developed and shared across all disciplines. As the science advances, we must continue to maximize optimal recurrence detection in the right patients, keeping in mind the broader implications of value-based clinical decision-making, survivorship care and multidisciplinary patient management.

\section{Future perspective}

As melanoma care evolves over the next decade, there will be a continued increasing focus on individualized patient care. In particular, surveillance will be guided by the totality of estimated disease risk, which will include family and patient history, clinicopathologic tumor features and molecular assessments of tumor biology. In parallel, melanoma therapies will likely continue to advance and new best practices (i.e., surgical, pharmaceutical) will be implemented in the context of traditional ones, all of which must also be navigated with respect to individual risk, clinical situation and patient needs. It is likely that patients who have been treated for melanoma will live longer and appropriate immediate- and long-term surveillance will have to balance likelihood of recurrence, benefit of early detection for therapeutic intervention and patient reassurance versus burden as a part of disease management and ongoing survivorship. Likewise, increasing scrutiny of healthcare expenditures will guide medical decisions in regards to surveillance. Thus, individualized patient assessment of recurrence will be utilized to decrease costs of unnecessary and dangerous testing of patients unlikely to recur. Over the next decade, these concepts should and will be better outlined and delineated in clinical guidelines. 
any organization or entity with a financial interest in or financial conflict with the subject matter or materials discussed in the manuscript apart from those disclosed.

No funded writing assistance was utilized in the production of this manuscript.

\section{Open access}

This work is licensed under the Attribution-NonCommercial-NoDerivatives 4.0 Unported License. To view a copy of this license, visit http://creativecommons.org/licenses/by-nc-nd/4.0/

\section{Acknowledgment}

K Plasseraud an employee and options holder at Castle Biosciences, Inc. provided writing assistance for this review.

\section{Executive summary}

Surveillance in contemporary melanoma management

- Adjuvant treatments and therapies for metastatic melanoma have been shown to improve outcomes, are recommended in national guidelines and have now been incorporated in the clinical management of melanoma patients.

- Given these therapeutic advances, early detection of recurrences has become more relevant; however, guidelines for surveillance imaging remain broad and do not recommend any imaging for earlier stage patients.

Benefits of early detection on curative-intent resections \& survival

- A large, prospective study demonstrated that early detection of melanoma recurrences may have a survival benefit, even accounting for lead-time bias.

- In a retrospective study of Stage III-V patients who had positron emission tomography (PET)/computed tomography (CT) scans, imaging detected $60 \%$ of recurrences and the majority of these were treated with curative intent surgery.

- Another study of Stage III patients found that the majority of the recurrences were imaging-detected and approximately half were treated with curative intent surgery.

Efficacy of routine cross-sectional imaging in detecting asymptomatic distant recurrences

- In a prospective, 10-year study of Stage IIB-III patients, $84 \%$ of distant metastases were detected by imaging, primarily CT scans and $92 \%$ were asymptomatic.

- In a retrospective study of high-risk Stage II-IV patients in clinical trials who underwent routine CT scanning, 75\% of distant recurrences, all asymptomatic, were detected by imaging.

- In a retrospective study of Stage IIIB-C patients who had CT scans and brain magnetic resonance images, two-thirds of recurrences were detected upon imaging.

- Despite not showing a direct link between surveillance and survival, other studies have shown $>90 \%$ sensitivity of imaging for distant metastasis detection.

\section{Patient detection versus imaging}

- Patients or physicians have been reported to detect the majority of melanoma recurrences; however some recurrences (skin, nodal) are more likely to be detected by physical examination, while distant metastases are more likely to be detected by imaging.

- The efficacy of imaging is difficult to evaluate if routine imaging was not employed or was unknown.

- Despite this, even in studies without routine imaging, imaging helped identify distant metastases, suggesting a combination of patient, physician and imaging detection is important.

The role for imaging in early identification of metastatic disease to improve patient outcomes

- Emerging evidence suggests that low metastatic tumor burden may have an improved response to contemporary treatments and curative-intent surgery, underscoring the need for early detection of disease recurrence or progression.

Targeting surveillance imaging toward high-risk melanoma patients

- Intensive routine imaging should not be applied universally to all patients with melanoma as this is not beneficial or cost-effective.

- Rather, accurate risk stratification is crucial to target surveillance imaging to high-risk patients.

Future directions for surveillance \& survivorship

- Genetic profiling for predicting metastatic risk is used in clinical practice in cutaneous melanoma, as well as uveal melanoma and other cancers; molecular analysis may contribute to a comprehensive risk assessment tool to help guide surveillance.

- As more patients are diagnosed and living with melanoma, the development of risk-appropriate surveillance imaging protocols is important for value-based medicine and survivorship. 


\section{References}

Papers of special note have been highlighted as: $\bullet$ of interest; $\bullet \bullet$ of considerable interest

1. Salama AK, De Rosa N, Scheri RP et al. Hazard-rate analysis and patterns of recurrence in early stage melanoma: moving towards a rationally designed surveillance strategy. PLoS ONE 8(3), e57665 (2013).

2. Bhutiani N, Egger ME, Mcmasters KM. Optimizing follow-up assessment of patients with cutaneous melanoma. Ann. Surg. Onc. 24(4), 861-863 (2017).

3. Swetter SM. Commentary: Improved patient outcomes remain elusive after intensive imaging surveillance for high-risk melanoma. $J$. Am. Acad. Dermatol. 75(3), 525-527 (2016).

4. Trotter SC, Sroa N, Winkelmann RR, Olencki T, Bechtel M. A global review of melanoma follow-up guidelines. J. Clin. Aesthet. Dermatol. 6(9), 18-26 (2013).

- Comprehensive comparison of melanoma follow-up across international guidelines.

5. Dummer R, Hauschild A, Lindenblatt N, Pentheroudakis G, Keilholz U, Committee EG. Cutaneous melanoma: ESMO Clinical Practice Guidelines for diagnosis, treatment and follow-up. Ann. Oncol. 26(Suppl. 5), v126-132 (2015).

6. Macbeth F, Newton-Bishop J, O'Connell S, Hawkins JE, Guideline Development G. Melanoma: summary of NICE guidance. BMJ. 351, h3708 (2015).

7. Swetter SM, Tsao H, Bichakjian CK et al. Guidelines of care for the management of primary cutaneous melanoma. J. Am. Acad. Dermatol. 80(1), 208-250 (2019).

8. Coit DG, Thompson JA, Albertini MR et al. Melanoma, Version 1.2019, NCCN Clinical Practice Guidelines in Oncology. (2019).

9. Xing Y, Cromwell KD, Cormier JN. Review of diagnostic imaging modalities for the surveillance of melanoma patients. Dermatol. Res. Pract. 2012, 941921 (2012).

10. Balch CM, Gershenwald JE, Soong SJ et al. Final version of 2009 AJCC melanoma staging and classification. J. Clin. Oncol. 27(36), 6199-6206 (2009).

11. Rockberg J, Amelio JM, Taylor A, Jorgensen L, Ragnhammar P, Hansson J. Epidemiology of cutaneous melanoma in Sweden-Stage-specific survival and rate of recurrence. Int. J. Cancer 139(12), 2722-2729 (2016).

12. Joseph RW, Elassaiss-Schaap J, Wolchok JD et al. Baseline tumor size as an independent prognostic factor for overall survival in patients with metastatic mealnoma treated with the anti-PD1 monoclonal antibody MK-3475. J. Clin. Oncol. 32(15_suppl.), 3015 (2014).

13. Kaufman H, Amatruda T, Nemunaitis JJ et al. Tumor size and clinical outcomes in melanoma patients (MEL pts) treated with talimogene laherparepvec (T-VEC). J. Clin. Oncol. 33(15_suppl.), 9074 (2015).

14. Long GV, Flaherty KT, Stroyakovskiy D et al. Dabrafenib plus trametinib versus dabrafenib monotherapy in patients with metastatic BRAF V600E/K-mutant melanoma: long-term survival and safety analysis of a phase 3 study. Ann. Oncol. 28(7), 1631-1639 (2017).

15. Menzies AM, Haydu LE, Carlino MS et al. Inter- and intra-patient heterogeneity of response and progression to targeted therapy in metastatic melanoma. PLoS ONE 9(1), e85004 (2014).

16. Nishino M, Giobbie-Hurder A, Ramaiya NH, Hodi FS. Response assessment in metastatic melanoma treated with ipilimumab and bevacizumab: CT tumor size and density as markers for response and outcome. J. Immunother. Cancer 2(1), 40 (2014).

17. Ribas A, Hamid O, Daud A et al. Association of pembrolizumab with tumor response and survival among patients with advanced melanoma. JAMA 315(15), 1600-1609 (2016).

18. Koskivuo I, Kemppainen J, Giordano $S$ et al. Whole body PET/CT in the follow-up of asymptomatic patients with stage IIB-IIIB cutaneous melanoma. Acta Oncol. 55(11), 1355-1359 (2016).

19. Garbe C, Paul A, Kohler-Spath H et al. Prospective evaluation of a follow-up schedule in cutaneous melanoma patients: recommendations for an effective follow-up strategy. J. Clin. Oncol. 21(3), 520-529 (2003).

20. Leiter U, Buettner PG, Eigentler TK, Forschner A, Meier F, Garbe C. Is detection of melanoma metastasis during surveillance in an early phase of development associated with a survival benefit? Melanoma Res. 20(3), 240-246 (2010).

-• Long-term follow-up with overall survival time adjusted for lead-time bias of study described in Garbe et al. [19]; the study showed earlier detected recurrences are associated with improved overall survival rates compared with those detected later.

21. Leon-Ferre RA, Kottschade LA, Block MS et al. Association between the use of surveillance PET/CT and the detection of potentially salvageable occult recurrences among patients with resected high-risk melanoma. Melanoma Res. 27(4), 335-341 (2017).

22. Lewin J, Sayers L, Kee D et al. Surveillance imaging with FDG-PET/CT in the post-operative follow-up of stage 3 melanoma. Ann. Oncol. 29(7), 1569-1574 (2018).

23. Podlipnik S, Carrera C, Sanchez M et al. Performance of diagnostic tests in an intensive follow-up protocol for patients with American Joint Committee on Cancer (AJCC) stage IIB, IIC, and III localized primary melanoma: a prospective cohort study. J. Am. Acad. Dermatol. 75(3), 516-524 (2016).

-. Prospective study evaluating the detection of recurrences by a routine imaging protocol in high-risk melanoma patients; most distant recurrences were detected asympomatically by imaging. 
24. Park TS, Phan GQ, Yang JC et al. Routine computer tomography imaging for the detection of recurrences in high-risk melanoma patients. Ann. Surg. Oncol. 24(4), 947-951 (2017).

-• Retrospective study at the National Cancer Institute of high-risk melanoma patients undergoing routine surveillance in vaccine therapy clinical trials, evaluating recurrence detection.

25. Lim KHJ, Spain L, Barker C et al. Contemporary outcomes from the use of regular imaging to detect relapse in high-risk cutaneous melanoma. ESMO Open. 3(2), e000317 (2018).

26. Livingstone E, Krajewski C, Eigentler TK et al. Prospective evaluation of follow-up in melanoma patients in Germany - results of a multicentre and longitudinal study. Eur. J. Cancer 51(5), 653-667 (2015).

27. Madu MF, Timmerman P, Wouters M, Van Der Hiel B, Van Der Hage JA, Van Akkooi ACJ. PET/CT surveillance detects asymptomatic recurrences in stage IIIB and IIIC melanoma patients: a prospective cohort study. Melanoma Res. 27(3), 251-257 (2017).

28. Berger AC, Ollila DW, Christopher A et al. Patient symptoms are the most frequent indicators of recurrence in patients with American Joint Committee on Cancer Stage II melanoma. J. Am. Coll. Surg. 224(4), 652-659 (2017).

29. Lee AY, Droppelmann N, Panageas KS et al. Patterns and timing of initial relapse in pathologic stage II melanoma patients. Ann. Surg. Oncol. 24(4), 939-946 (2017).

30. Romano E, Scordo M, Dusza SW, Coit DG, Chapman PB. Site and timing of first relapse in stage III melanoma patients: implications for follow-up guidelines. J. Clin. Oncol. 28(18), 3042-3047 (2010).

31. Lyle MK, Lee JHJ, Menzies AM et al. Lesion-specific patterns of response and progression with anti-PD1 treatment in metastatic melanoma (MM). J. Clin. Oncol. 32(15_suppl), 9077 (2014).

32. Weber J, Mandala M, Del Vecchio M et al. Adjuvant nivolumab versus ipilimumab in resected stage III or IV Melanoma. NEJM 377(19), 1824-1835 (2017).

33. Schadendorf D, Long GV, Stroiakovski D et al. Three-year pooled analysis of factors associated with clinical outcomes across dabrafenib and trametinib combination therapy phase 3 randomised trials. Eur. J. Cancer 82, 45-55 (2017).

34. Long GV, Grob JJ, Nathan P et al. Factors predictive of response, disease progression, and overall survival after dabrafenib and trametinib combination treatment: a pooled analysis of individual patient data from randomised trials. Lancet Oncol. 17(12), 1743-1754 (2016).

35. Maio M, Lewis K, Demidov L et al. Adjuvant vemurafenib in resected, BRAF V600 mutation-positive melanoma (BRIM8): a randomised, double-blind, placebo-controlled, multicentre, Phase 3 trial. Lancet Oncol. 19(4), 510-520 (2018).

36. Sayers L, Lewin J, Kee D et al. Surveillance imaging with FDG-PET/CT in the post-operative follow-up of stage 3 melanoma. J. Clin. Oncol. 35(Suppl.), 9563 (2017).

37. Vogel RI, Strayer LG, Ahmed RL, Blaes A, Lazovich D. A qualitative study of quality of life concerns following a melanoma diagnosis. J. Skin Cancer. 2017, 2041872 (2017).

38. Stamataki Z, Brunton L, Lorigan P, Green AC, Newton-Bishop J, Molassiotis A. Assessing the impact of diagnosis and the related supportive care needs in patients with cutaneous melanoma. Support. Care Cancer 23(3), 779-789 (2015).

39. Rychetnik L, Mccaffery K, Morton R, Irwig L. Psychosocial aspects of post-treatment follow-up for stage I/II melanoma: a systematic review of the literature. Psychooncology 22(4), 721-736 (2013).

40. Bastiaannet E, Hoekstra-Weebers JE, Francken AB, Jager PL, Van Der Jagt EJ, Hoekstra HJ. Perception of burden experienced during diagnostic tests by melanoma patients with lymph node metastases. Melanoma Res. 19(1), 36-41 (2009).

41. Adler NR, Kelly JW, Guitera P et al. Methods of melanoma detection and of skin monitoring for individuals at high risk of melanoma: new Australian clinical practice. Med. J. Aust. 210(1), 41-47 (2019).

42. Coit D, Thompson JA, Albertini M et al. Uveal Melanoma NCCN Guidelines National Comprehensive Cancer Network v1.2018 (2018).

43. Aaberg TM, Jr., Cook RW, Oelschlager K, Maetzold D, Rao PK, Mason JO, 3rd. Current clinical practice: differential management of uveal melanoma in the era of molecular tumor analyses. Clin. Ophthalmol. 8, 2449-2460 (2014).

44. Plasseraud KM, Cook RW, Tsai T et al. Clinical performance and management outcomes with the decisionDx-UM gene expression profile test in a prospective multicenter study. J. Oncol. 2016, 5325762 (2016).

45. Schuitevoerder D, Heath M, Cook RW et al. Impact of gene expression profiling on decision-making in clinically node negative melanoma patients after surgical staging. J. Drugs Dermatol. 17(2), 196-199 (2018).

46. Berger AC, Davidson RS, Poitras JK et al. Clinical impact of a 31-gene expression profile test for cutaneous melanoma in 156 prospectively and consecutively tested patients. Curr. Med. Res. Opin. 32(9), 1599-1604 (2016).

47. Dillon LD, Gadzia JE, Davidson RS et al. Prospective, multicenter clinical impact evaluation of a 31-gene expression profile test for management of melanoma patients. SKIN 2(2), 111-121 (2018).

48. Farberg AS, Glazer AM, White R, Rigel DS. Impact of a 31-gene expression profiling test for cutaneous melanoma on dermatologists' clinical management decisions. J. Drugs. Derm. 16(5), 428-431 (2017).

49. Greenhaw BN, Zitelli JA, Brodland DG. Estimation of prognosis in invasive cutaneous melanoma: an independent study of the accuracy of a gene expression profile test. Dermatol. Surg. 44(12), 1494-1500 (2018). 
50. Ferris LK, Farberg AS, Middlebrook B et al. Identification of high-risk cutaneous melanoma tumors is improved when combining the online American Joint Committee on Cancer Individualized Melanoma Patient Outcome Prediction Tool with a 31-gene expression profile-based classification. J. Am. Acad. Derm. 76(5), 818-825.e3 (2017).

51. Gerami P, Cook RW, Russell MC et al. Gene expression profiling for molecular staging of cutaneous melanoma in patients undergoing sentinel lymph node biopsy. J. Am. Acad. Derm. 72(5), 780-785.e783 (2015).

52. Gerami P, Cook RW, Wilkinson J et al. Development of a prognostic genetic signature to predict the metastatic risk associated with cutaneous melanoma. Clin. Cancer Res. 21(1), 175-183 (2015).

53. Zager JS, Gastman BR, Leachman S et al. Performance of a prognostic 31-gene expression profile in an independent cohort of 523 cutaneous melanoma patients. BMC Cancer 18(1), 130 (2018).

54. Hsueh EC, Debloom JR, Lee J et al. Interim analysis of survival in a prospective, multi-center registry cohort of cutaneous melanoma tested with a prognostic 31-gene expression profile test. J. Hematol. Oncol. 10(1), 152 (2017).

55. Long GV, Menzies AM, Nagrial AM et al. Prognostic and clinicopathologic associations of oncogenic BRAF in metastatic melanoma. J. Clin. Oncol. 29(10), 1239-1246 (2011).

56. Ekedahl $\mathrm{H}$, Cirenajwis $\mathrm{H}$, Harbst $\mathrm{K}$ et al. The clinical significance of BRAF and NRAS mutations in a clinic-based metastatic melanoma cohort. Br. J. Dermatol. 169(5), 1049-1055 (2013).

57. Brobeil A, Rapaport D, Wells K et al. Multiple primary melanomas: implications for screening and follow-up programs for melanoma. Ann. Surg. Oncol. 4(1), 19-23 (1997).

58. Podlipnik S, Moreno-Ramirez D, Carrera C et al. Cost-effectiveness analysis of imaging strategy for an intensive follow-up of patients with AJCC stage IIB, IIC and III malignant melanoma. Br. J. Dermatol. doi:10.1111/bjd.16833 (2018).

- Cost analysis of prospective imaging study described in [23] to detected recurrences in high-risk melanoma patients. 
